\title{
Förderpreis für Sportdidaktik 2004
}

Der Förderpreis für Sportdidaktik (500 Euro) soll 2004 zum siebten Mal vergeben werden. Ausgezeichnet werden deutschsprachige Arbeiten, die nicht älter als zwei Jahre sind und praxisbezogen die Erziehungswirkungen und -möglichkeiten des Sportunterrichts untersuchen sowie dazu beitragen, Defizite aufzuzeigen, sie auszugleichen oder die Erziehungsaufgabe besser zu erfüllen.

Die Arbeiten sollen bis zum 31. 11. 2003 beim Lehrstuhl für Sportpädagogik, Regensburger Straße 160, 90478 Nürnberg, eingereicht werden. Sie sollen ein selbstgewähltes Kennwort tragen und in einem verschlossenen Umschlag Namen, Anschrift und Kennwort des Verfassers enthalten. Weitere Auskünfte erhalten Interessenten von Frau Irmgard Rupp (Lehrstuhl für Sportpädagogik; Anschrift wie oben).

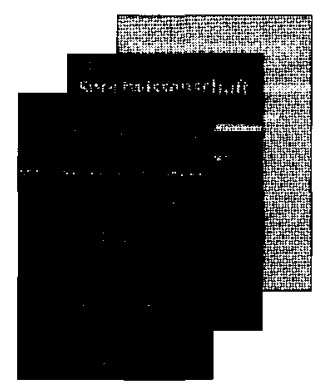

\section{Vorschau}

Folgende Beiträge wurden nach Prüfung durch die Gutachterinnen und Gutachter zur Publikation angenommen und stehen zur Veröffentlichung in den nächsten Heften an

Jürgen Baur/

Ulrike Burrmann

Lutz Schega/

Christine Stucke

Petra Wagner Roland Singer
Jugendliche Sportvereinsmitglieder als „Trittbrettfahrer“?

Belastung und Beanspruchung als Einflussgrößen der Intensitätssteuerung im Rehabilitations- und Behindertensport

Ein Fragebogen zur Erfassung der habituellen körperlichen Aktivität verschiedener Bevölkerungsgruppen 